\title{
An Environmental Control and Life Support System Concept for a Pressurized Lunar Rover
}

\author{
Robert M. Bagdigian ${ }^{1}$ \\ NASA Marshall Space Flight Center, Huntsville, AL, 35812 \\ and \\ Imelda Stambaugh ${ }^{2}$ \\ NASA Johnson Space Center, Houston, TX, 77058
}

\section{Introduction}

$\mathrm{P}$ ressurized rovers can add many attractive capabilities to a human lunar exploration campaign, most notably by extending the reach of astronauts far beyond the immediate vicinities of lunar landers and fixed assets such as habitats. Effective campaigns will depend on an efficient allocation of environmental control and life support system (ECLSS) equipment amongst mobile rovers and fixed habitats such that widespread and sustainable exploration can be achieved. This paper will describe some of the key drivers that influence the design of an ECLSS for a pressurized lunar rover and a conceptual design that has been formulated to address those drivers. Opportunities to realize programmatic and operational efficiencies through commonality of rover ECLSS and extravehicular activity (EVA) equipment have also been explored and will be described. Plans for the inclusion of ECLSS functionality in prototype lunar rovers will be summarized.

\section{Human Lunar Exploration Campaign Concepts}

Cooperating under the auspices of the International Space Exploration Coordination Group (ISECG), representatives of the International Architecture Working Group (IAWG) and International Objectives Working Group (IOWG) have been developing and comparing conceptual approaches to establish and sustain an integrated human lunar exploration campaign in ways that fulfill the multilateral objectives of the participating countries. A number of approaches were conceived and evaluated relative to a variety of figures of merit including scientific return, extensibility of the approaches to other exploration missions, and promoting public engagement. From these studies, a Global Point of Departure (GPoD) has been formulated to serve as a framework to support international planning and negotiations leading to an eventual international human lunar exploration campaign. A key characteristic of the GPoD campaign is its phased approach. Initial lunar robotic precursor missions increase knowledge, test hardware, and reduce risk. Subsequent short duration human missions to a lunar pole will provide opportunities for both local polar exploration and to validate and verify critical mobility and power infrastructure assets. The extent of lunar exploration is then increased to near-polar and distant non-polar locations by relocating key assets via mobility. Discoveries and lessons learned acquired in this manner are then used to support the site selection and optimization of fixed assets capable of enabling extended human stays of at least 60 days and development of Mars-forward technologies. Intermingling short duration human sortie missions to other locations throughout the campaign are accommodated to meet science objectives as required.

One of the lasting lessons of the Apollo program that influenced the GPoD architecture is that surface mobility is key to improving the efficiency of humans on the lunar surface. To that end, NASA has been developing surface mobility vehicles essential to meeting many lunar science objectives. One such vehicle that is being evaluated is a Lunar Electric Rover (LER), or equivalent, a prototype of which is shown in Fig. 1. While smaller than a

\footnotetext{
${ }^{1}$ Chief, Environmental Control and Life Support Development Branch, Marshall Space Flight Center/ES62.

${ }^{2}$ Life Support Engineer, Thermal Systems and Engineering Support Branch, Johnson Space Center/EC2.
} 
pressurized habitat, a pressurized rover can offer the capability to extend the reach of astronauts far beyond a fixed

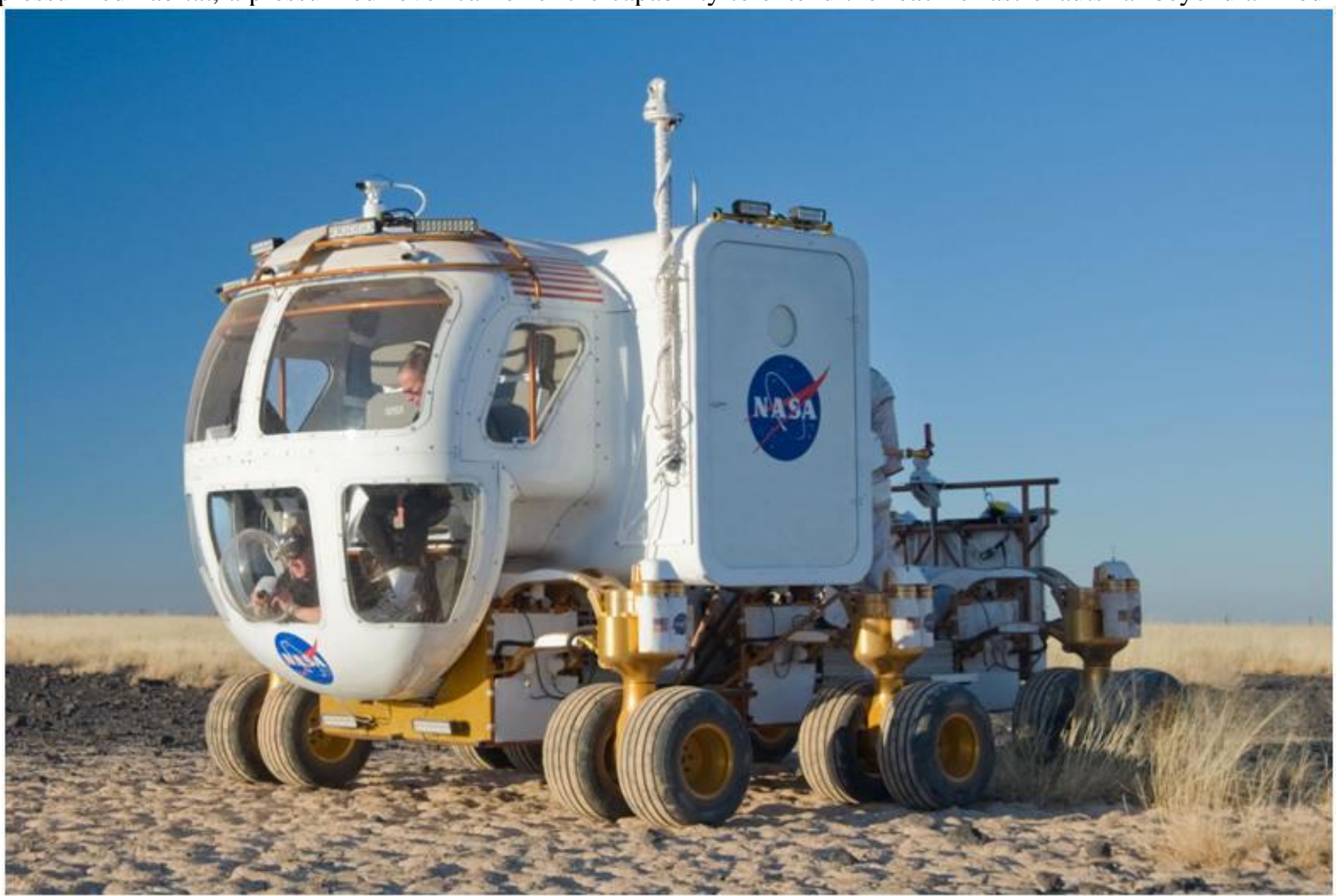

Figure 1. Prototype Lunar Electric Rover (LER)

lunar outpost location while retaining access to laboratory equipment available in the outpost for increased scientific return. Studies and field tests indicate that a two-person rover could be equipped to handle three-day excursions with its own on-board resources, while longer excursions lasting up to 14 days could be conducted with periodic resupply from transportable logistics carriers. By configuring the rover with exterior-mounted spacesuits that the crew could don quickly through a rear-entry hatch, and by controlling rover atmosphere conditions such that extended pre-breathe periods are not required before the crew embarks on Extra-Vehicular (EVA), the extent and efficiency of human surface exploration can be significantly enhanced. By reducing the operational overhead of exiting and re-entering the rover, multiple short duration EVAs can be performed within a single day, thereby increasing exploration flexibility to study widely dispersed lunar features as they are encountered.

The combination of short rover-based excursions with extended stays centered at a fixed outpost offers many attractive exploration features. However, the development and efficient deployment of environmental control and life support equipment throughout a lunar infrastructure that includes both fixed and mobile assets must address some unique technical and operational challenges. General considerations related to the development of an integrated ECLSS for a lunar outpost have been summarized previously (1). Given that an outpost will include some type of pressurized habitat(s) occupied by human crews for extended periods and that transportation costs will dictate that resupply rates be minimized to the lowest practical levels, the functions required of a lunar surface ECLSS can be defined with reasonable confidence. Those functions, along with the subsystems to which they may likely be assigned, are listed in Fig. 2. 

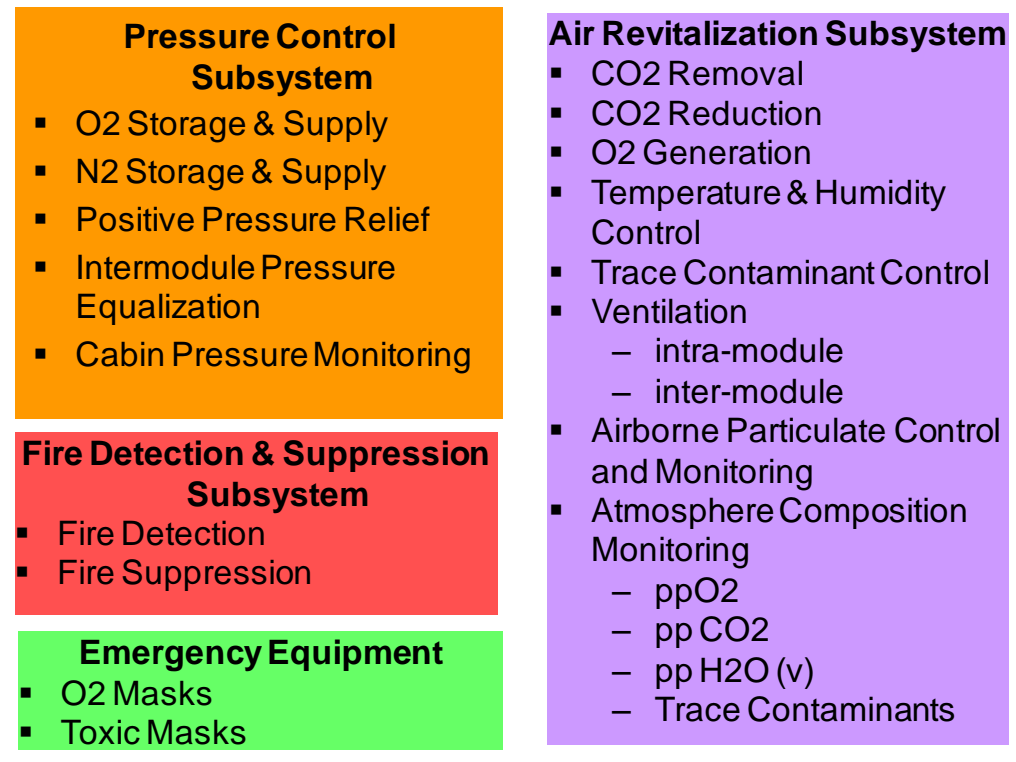
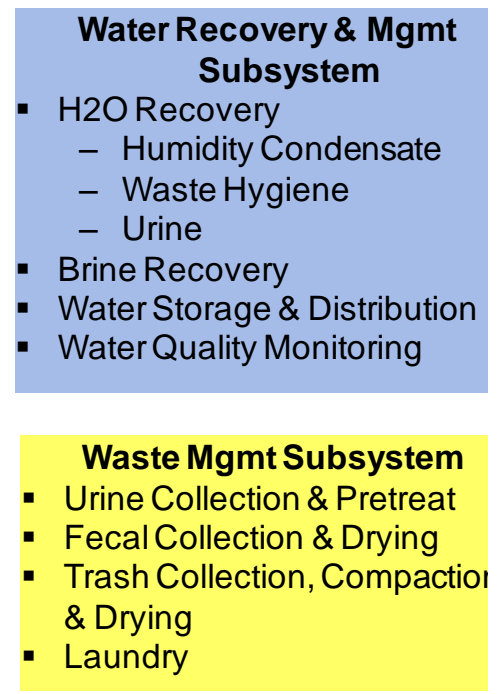

Figure 2. Typical Environmental Control and Life Support Functions

In a complete lunar outpost made up of pressurized habitat(s), logistics modules, and rovers, it will be necessary to distribute the ECLSS equipment needed to perform the functions in Fig. 2 among an array of pressurized elements in a manner that supports polar, non-polar exploration, and eventual outpost buildup while avoiding unnecessary duplication of equipment. Centralizing much of a lunar campaign's ECLSS within a minimum number of Pressurized Core Module(s) (PCM) can be an effective strategy in minimizing the amount of equipment that will be needed in volume- and resource-constrained pressurized rovers and in minimizing the tare weight of pressurized logistics modules. In such a strategy, inter-module ventilation can be used to maintain habitable conditions within attached pressurized elements. At the same time, extended crew occupancy of pressurized rovers operating remotely from the outpost dictates that a substantial amount of ECLSS functionality be provided independently in those rovers. A simplified depiction of how ECLSS functions might be strategically distributed among lunar pressurized elements is shown in Fig.3. The notional set of surface assets depicted in the figure includes PCMs, Pressurized Logistics Modules (PLM) and unpressurized logistics carriers, Portable Utility Pallets (PUP), LERs, In-Situ Resource Utilization (ISRU) systems, and Extra-Vehicular Activity (EVA) systems.

In the strategy depicted in Fig. 3, the PCM's comparatively large pressurized volume and the fact that (unlike a LER) it is not mass constrained for routine mobility makes it an ideal asset in which to centralize as much ECLSS equipment as possible. The PCM includes a complete complement of ECLSS equipment, including that which is specifically needed for sustainable lunar operations through the regeneration of water and oxygen supplies. Water recovery equipment provides for the recovery of potable water from a variety of wastewaters, including humidity condensate, urine, waste hygiene water, and water liberated from the drying of trash and fecal wastes. Oxygen recovery equipment provides for the recovery of oxygen from carbon dioxide through the electrolysis of water that is produced via reaction of carbon dioxide and hydrogen.

The LERs on the other hand are small and must be kept lightweight in order to maximize their excursion range for a given amount of on-board energy storage. Therefore, the amount of ECLSS equipment allocated to LERs must be carefully considered and prioritized to that required to sustain crew on relatively short duration excursions away from a relatively more capable fixed lunar outpost. Current LER operations concepts envision them being normally operated by two crewmembers for up to three consecutive days without resupply, with two LERs typically exploring regions in tandem. Longer excursions are facilitated by the replenishment of oxygen and water consumables from logistics carriers such as PUPs that are strategically placed by the LERs at central locations suitable to support a exploration at targeted localities within a broader lunar region. During excursions, crewmember inter-vehicular activity (IVA) operations are enhanced by the capability to dock the two LERs together for crew and material exchanges. Similarly, LER docking with fixed PLMs facilitate IVA re-provisioning and docking with PCMs allows crew access to additional habitation, science, logistics, and maintenance capabilities.

From an ECLSS perspective, the operations that have the greatest influence on LER functionality requirements are excursions during which crewmembers need to be sustained while away from other lunar surface elements. The 
minimal ECLSS functionality required in an LER includes carbon dioxide removal, trace contaminant control, humidity control, atmosphere monitoring, and temperature control all facilitated by cabin ventilation. Cabin pressure control is maintained with oxygen and nitrogen gases supplied from on-board tanks sized to support three nominal days with two crewmembers plus a contingency fourth day with four crewmembers. Similarly, on-board stored water supplies, waste management collection hardware, and storage equipment supports three nominal days plus a fourth contingency day. Fire detection and suppression and emergency response capabilities are included. Cabin and surface particulate management functions are also included, particularly to mitigate the accumulation of lunar dust within the habitable cabin. Re-provisioning of stored consumable gases (oxygen and nitrogen) and water is facilitated through interfaces to PCMs and PUPs.

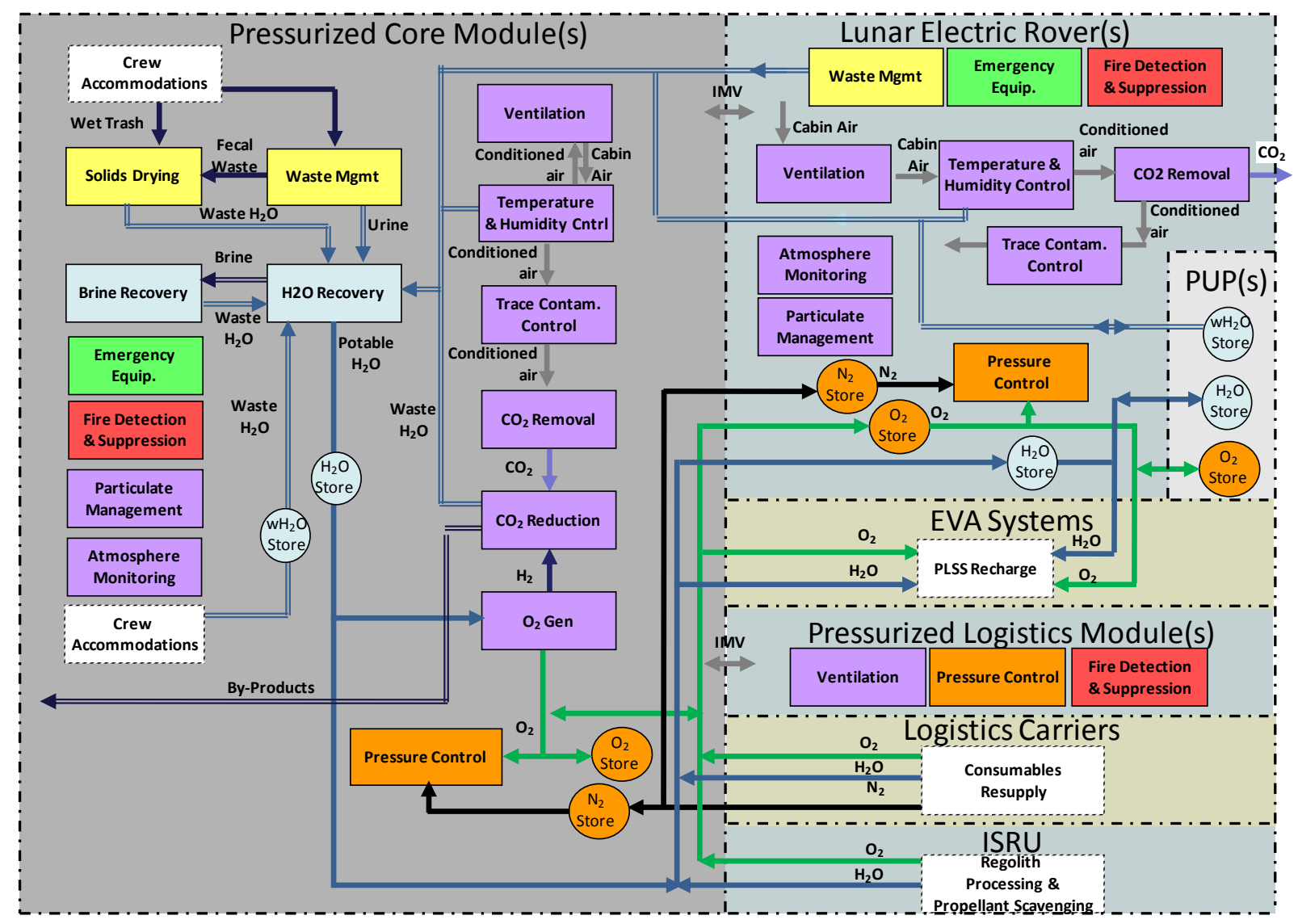

Figure 3. Example Distribution of ECLSS Equipment Throughout Lunar Outpost Elements

Although short duration LER excursions individually don't merit being burdened with the equipment and power to recycle oxygen and water, cumulative excursions can rather quickly reach a break-even point at which the sustainability of a lunar campaign as a whole would benefit from recovering oxygen and water from waste sources that are generated within LERs during individual excursions. For this reason, the capability to collect, stabilize, store, and return wastes (carbon dioxide, humidity condensate, urine, trash, and fecal material) generated in LERs on excursions to the fixed outpost for subsequent resource recovery are anticipated in campaign planning.

\section{Environmental Control and Life Support Concept for Lunar Pressurized Rover}

A conceptual LER ECLSS architecture to fulfill the functional allocation depicted graphically in Fig. 3 was developed as a tool to assess resource (mass, volume, power) requirements and support the development of excursion concepts of operations. The resulting conceptual LER ECLSS architecture is shown in Fig. 4.

Much of the conceptual LER ECLSS architecture is in support of cabin air revitalization. A cabin fan and ducting provide overall mixing of cabin atmosphere and transport of sensible and latent heat loads to a condensing heat exchanger. In the LER concept, vehicle heat rejection is provided by a radiator that is supplemented by a 
water-based fusible heat sink that provides thermal capacitance to accommodate transient peak loads. The cabin condensing heat exchanger rejects cabin sensible and latent heat loads to the radiator and heat sink system. A bypass around the condensing heat exchange facilitates the management of cabin temperatures and humidity. A fraction of the relatively dry air exiting the condensing heat exchanger is drawn into an air revitalization subsystem which includes carbon dioxide removal and trace contaminant control beds, along with dedicated redundant fan assemblies. Open and closed oxygen loop capabilities have both been considered. A closed-loop strategy would allow oxygen to be recovered from the $\mathrm{CO}_{2}$ via one of several possible technology pathways. Closed-loop strategies for LERs include deploying and operating oxygen loop closure equipment in the LERs themselves or compressing and storing $\mathrm{CO}_{2}$ on-board LERs and returning it to a centralized outpost or fixed facility for ultimate oxygen recovery. In light of today's state of the art in oxygen loop closure and $\mathrm{CO}_{2}$ compression technologies, lunar surface campaign strategies have been founded on the assumption that LERs would be outfitted with open oxygen loop architectures and the resulting oxygen resupply needs for lunar campaign scenarios have been accounted for accordingly. However, recovery of oxygen from the $\mathrm{CO}_{2}$ captured from LER cabin atmospheres could substantially increase the sustainability of lunar campaigns; technology advancements that would facilitate such a capability in resource-constrained vehicles such as LERs are areas worthy of technology development.

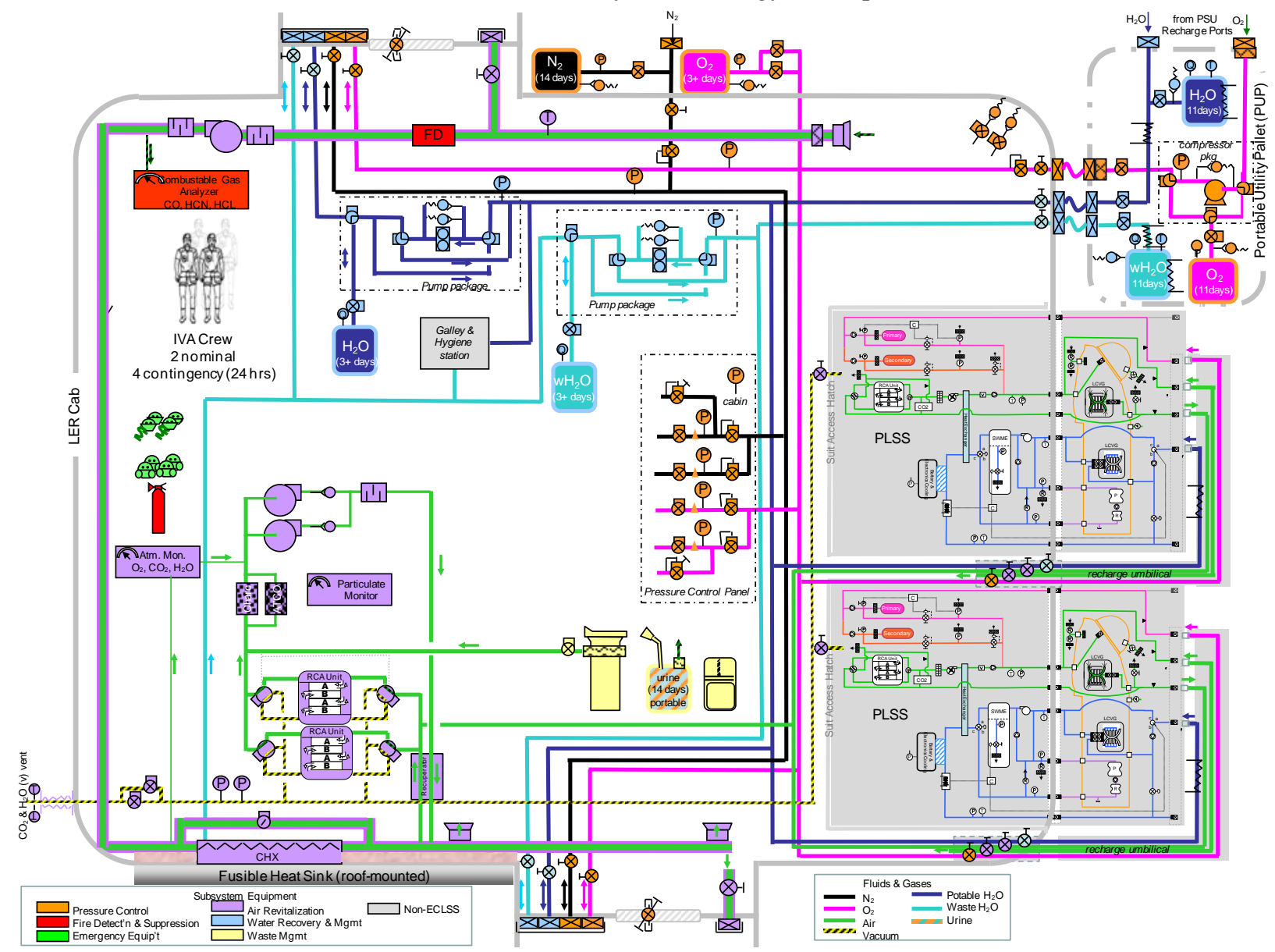

Figure 4. Conceptual ECLSS Architecture for a Lunar Electric Rover (LER)

Water losses associated with open-loop $\mathrm{CO}_{2}$ removal in LERs could be substantial over a lunar campaign life cycle. Therefore, the LER ECLSS concept includes a water-save feature that strips residual humidity from the slip stream leading to the $\mathrm{CO}_{2}$ removal beds and returns it to the cabin ventilation loop where it can be collected as liquid condensate from the cabin's condensing heat exchanger and ultimately returned to a central outpost for water recovery.

The LER air revitalization architecture concept includes provisions for atmosphere trace contaminant control via an expendable charcoal-based adsorption bed to control high molecular weight organics and a parallel ambient 
temperature catalytic oxidizer for methane control. An airborne particulate monitor is accounted for in the LER architecture concept in recognition of the high potential for lunar dust infiltration into the cabin environment during prolonged LER usage, even with advanced safeguards such as suitports designed to minimize such infiltration. An atmosphere monitor provides continuous measurement of cabin oxygen, carbon dioxide, and humidity levels.

The LER ECLSS architecture includes equipment dedicated to the control of LER cabin pressures and the storage and distribution of make-up gases. Oxygen and nitrogen are distributed to each LER docking port and to the PUP interface panel to facilitate the exchange of make-up gases between LERs, outpost elements, and deployed PUPs. Pressurized oxygen is also delivered to umbilical connections for charging primary and secondary oxygen storage bottles within the EVA Portable Life Support System (PLSS). Oxygen and nitrogen service panels provide redundant pressurization gas interfaces to the LER cabin. Oxygen and nitrogen storage tanks located outside the LER cabin pressure shell provide capacities to support three days of nominal usage by two crewmembers plus a 24hour supply for four crewmembers under emergency conditions. Pressure equalization valves on hatches facilitate LER docked operations and positive pressure relief assemblies protect against cabin over-pressurization.

Water is provided for crew consumption and basic hygiene needs. Potable and waste water (humidity condensate and waste hygiene water) are distributable to LER docking ports and PUP interfaces to facilitate exchanges in much the same fashion as oxygen and nitrogen. Waste water is collected and stored within the LER and PUPs for return to water recovery equipment in a centralized outpost or other fixed surface asset.

Waste management equipment provides collection, storage, stabilization, and odor control of urine, fecal waste, and trash. Urine, given its high long-term fouling potential, is not mixed with other waste water sources and is instead simply collected and stored in manually-transportable containers for crew exchange at the end of excursions. Similarly, fecal wastes are collected and stored in odor-controlled manually-transportable containers that are compatible with drying equipment in a centralized outpost. Trash collection, compaction, and odor control equipment is also included in the LER architecture concept.

Fire protection equipment included within the LER ECLSS architecture concept includes duct-mounted smoke detectors or other suitable bulk-cabin detector system, a contingency gas analyzer suitable to detect early combustion precursors, and a portable fire extinguisher. Personal protection equipment includes masks for post-fire conditions.

Equipment commonality will be an important contributor to the sustainability of any lunar exploration campaign. The LER ECLSS architecture concept highlights a number of candidate areas in which equipment common with other lunar campaign elements such as PCMs, PLMs, the Orion Crew Module, the Altair crew lander, EVA PLSS, and ISRU plants. Notable candidates include $\mathrm{CO}_{2}$ removal beds; preliminary sizing studies show that swing-beds sized for application within the EVA PLSS could also meet nominal and emergency $\mathrm{CO}_{2}$ removal needs within the LER cabin. Utilizing common swing bed designs in both the LER cabin and EVA PLSS can provide attractive programmatic savings by reducing development and life-cycle sparing costs and increasing mission flexibility. Controlling LER cabin $\mathrm{CO}_{2}$ levels through operation of EVA PLSS swing beds might also be viable, although integration and operational challenges would be greater. Other notable LER equipment candidates with high commonality potential include the atmosphere and particulate monitors, contingency gas analyzers and smoke detectors, portable fire extinguisher, personal protection equipment, fans, pumps, and a host of ancillary components such as valves and instrumentation.

\section{ECLSS Concepts for Rover Prototypes}

The Lunar Electric Rover (LER) project was developed by NASA to demonstrate and test technologies which progress the maturity of the rover design. LER has staggered ECLSS functionality in its current and future vehicle generations. There are three generations of the vehicle named the generation 1, generation 2 and generation 3 vehicles. The generation 1 LER contains an ECLSS which can be compared to that on a recreational vehicle. A compact air conditioning unit is used to simulate the Air Revitalization Subsystem (ARS), a camping style water tank and heater to dispense potable water for the Potable Water Subystem (PWS), trash container and camping style toilet for the Waste Management Subsystem (WMS), and a fire detector and no suppression device in the cabin for the Fire Detection and Suppression Subsystem (FDSS). No Pressure Control Subsystem (PCS) was part of this design. An estimate of the volume of ECLSS hardware in a Preliminary Design Review (PDR) level vehicle 
prototype was modeled in Computer Aided Design (CAD) and foam board to determine the ECLSS footprint in the vehicle. The generation 2 rover will improve on generation 1 design. The seal on the vehicle will be as air tight as possible and the vehicle shell will be built to pressure vessel code but not tested to verify the vessel integrity. The LER will have a higher capacity compact air conditioning unit to simulate the ARS, an improved potable water system design for the PWS, an improved trash containment system and camping style toilet for the WCS, a fire detector and suppression device for the FDSS. No PCS will be part of this design. Once again, an estimate of the volume of ECLSS hardware in a PDR level ECLSS prototype will be modeled in Computer Aided Design (CAD) and foam board to determine the ECLSS footprint in the vehicle. The generation 3 LER will be the closest prototype vehicle to the flight vehicle. It is anticipated that this vehicle will be a pressure vessel outfitted with PDR level ECLSS prototype hardware and be capable of vacuum chamber testing. This vehicle will contain a functioning PCS, PWS, ARS, WCS, and FDSS and will be utilized in field tests to demonstrate operational concepts and strategies for lunar surface exploration.

\section{Acronyms}

$\begin{array}{ll}A R S & =\text { Air Revitalization Subsystem } \\ C O_{2} & =\text { Carbon Dioxide } \\ E C L S S & =\text { Environmental Control and Life Support System } \\ E V A & =\text { Extra-Vehicular Activity } \\ F D S S & =\text { Fire Detection and Suppression Subsystem } \\ G P O D & =\text { Global Point of Departure } \\ I A W G & =\text { International Architecture Working Group } \\ I O W G & =\text { International Objectives Working Group } \\ I S E C G & =\text { International Space Exploration Coordination Group } \\ I S R U & =\text { In-Situ Resource Utilization } \\ I V A & =\text { Intra-Vehicular Activity } \\ L E R & =\text { Lunar Electric Vehicle } \\ P C M & =\text { Pressurized Core Module } \\ \text { PCS } & =\text { Pressure Control Subsystem } \\ \text { PLM } & =\text { Pressurized Logistics Module } \\ \text { PLSS } & =\text { Portable Life Support System } \\ \text { PDR } & =\text { Preliminary Design } \\ \text { PUP } & =\text { Portable Utility Pallet } \\ \text { PWS } & =\text { Potable Water Subsystem } \\ \mathrm{O}_{2} & =\text { Oxygen } \\ \text { WMS } & =\text { Waste Management Subsystem }\end{array}$

\section{Acknowledgments}

The authors would like to thank Walter Vonau and Kevin Lange of Jacobs Technology at the NASA Johnson Space Center for their contributions to technical trade studies that informed the evolution of the LER ECLSS architecture concept.

\section{References}

${ }^{1}$ Bagdigian, Robert M., "Challenges with Deploying and Integrating Environmental Control and Life Support Functions in a Lunar Architecture with High Degrees of Mobility," International Conference on Environmental Systems, 2009-01-2481, SAE, Savannah, GA, 2009. 